\title{
Redox oxides-based solar thermochemistry and its materialization to reactor/heat exchanger concepts for efficient solar energy harvesting, transformation and storage
}

Christos Agrafiotis, Martin Roeb, Christian Sattler Institute of Solar Research DLR/ Deutsches Zentrum für Luft- und Raumfahrt/ German Aerospace Center Linder Höhe, 51147 Köln, Germany
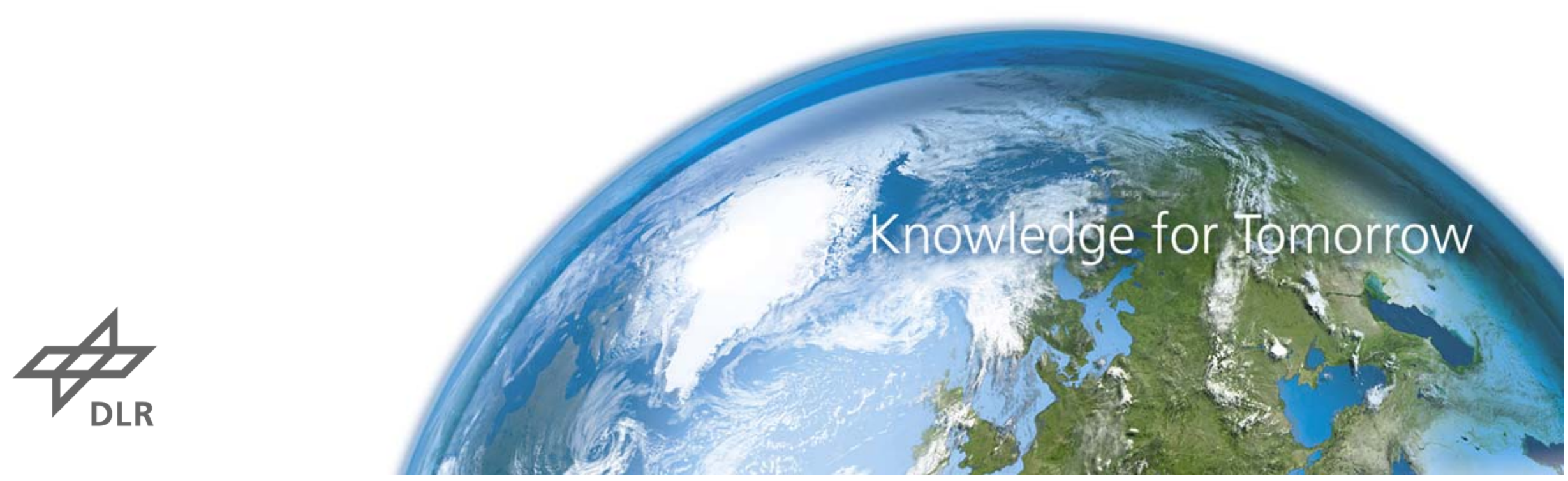


\section{Introduction}

- Solar fuels production from Concentrating Solar Systems and Solar Thermal Power Plants (STPPs)

- Solar fuels chemistries and reactors

- Commonalities in materials requirements and reactor concepts among solar energy conversion, storage and transformationrelated processes.

- Outlook, needs and ideas for the future.
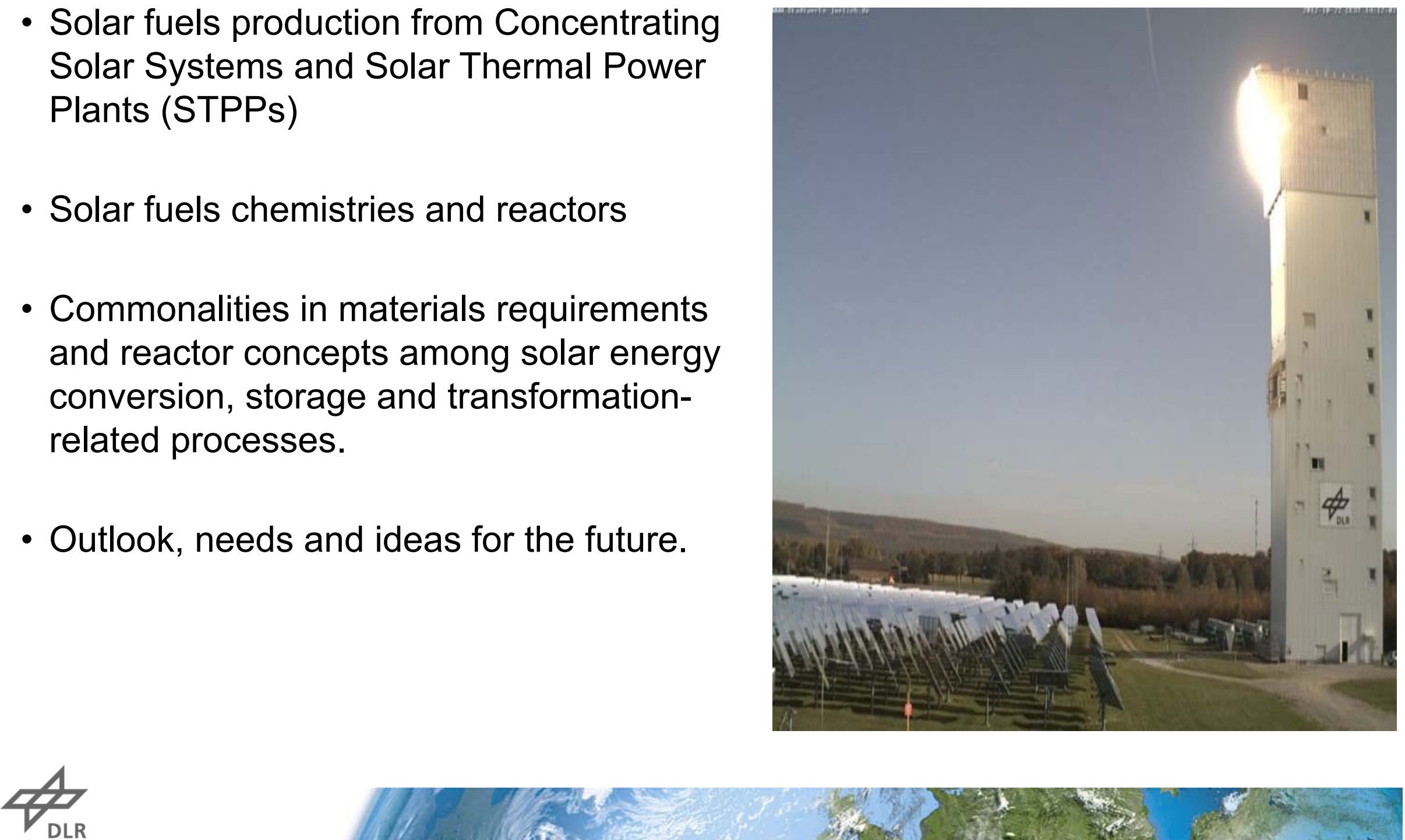


\section{Partial listing of various feedstocks and solar energy variances for solar liquid hydrocarbon fuels production}
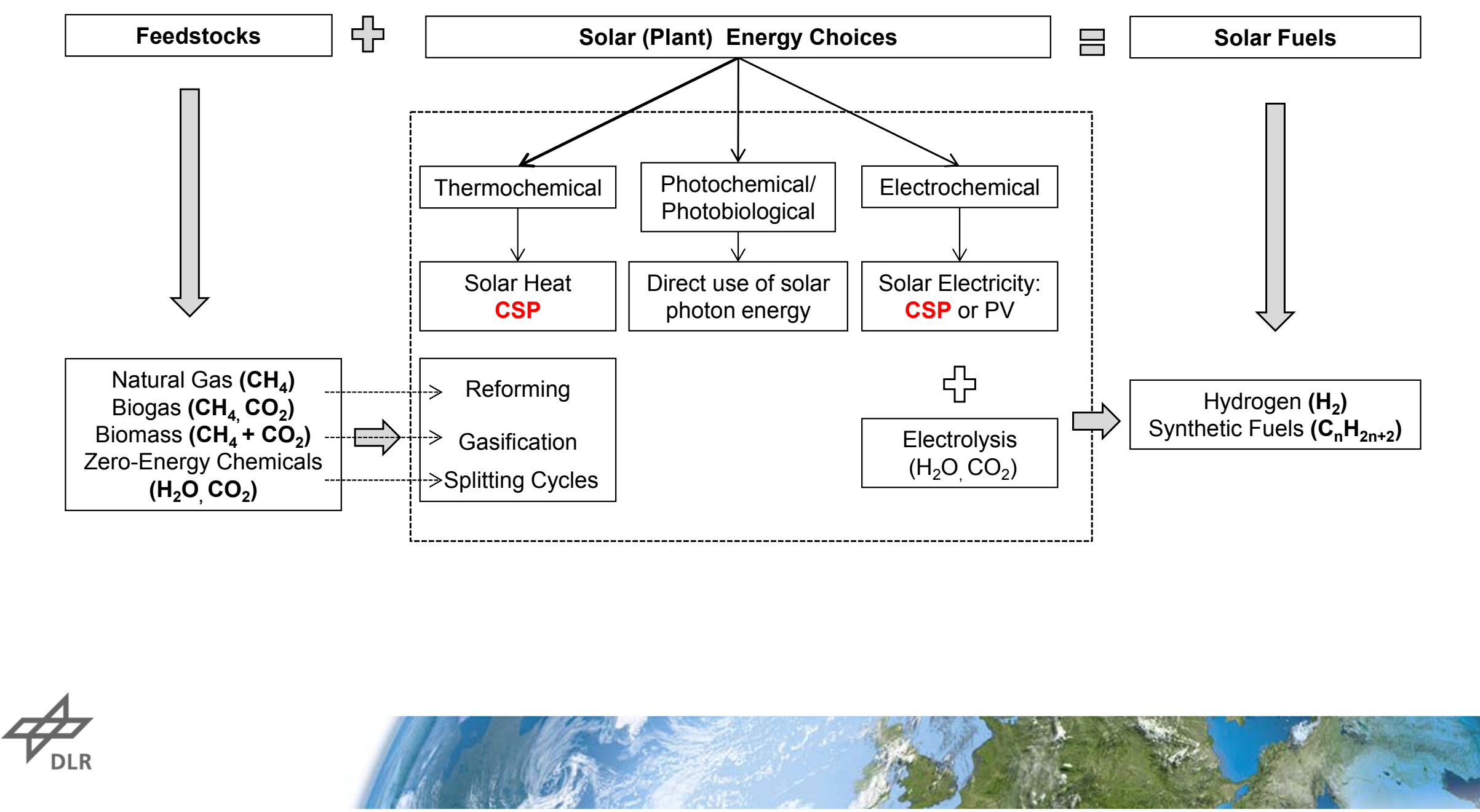


\section{Solar Methane Reforming- Reformer (heating) Technologies}

a) decoupled/allothermal

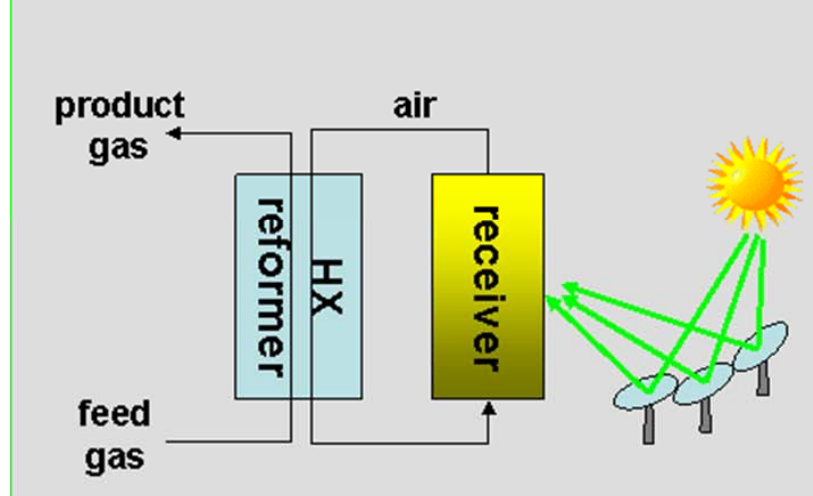

b) indirect (tube reactor)

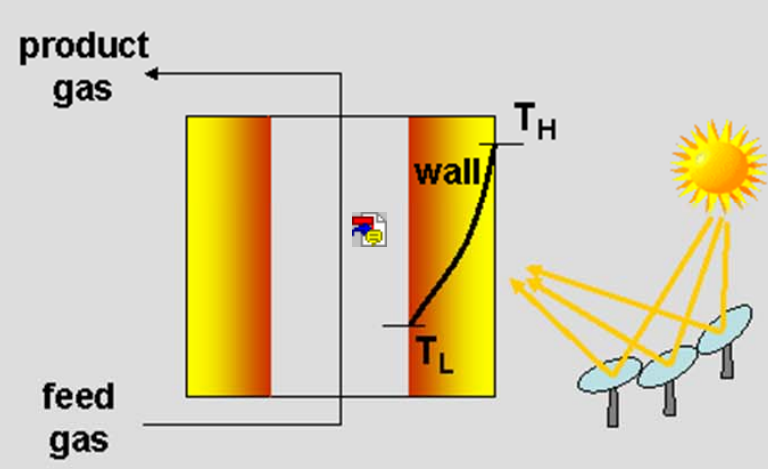

c) Integrated, direct, volumetric

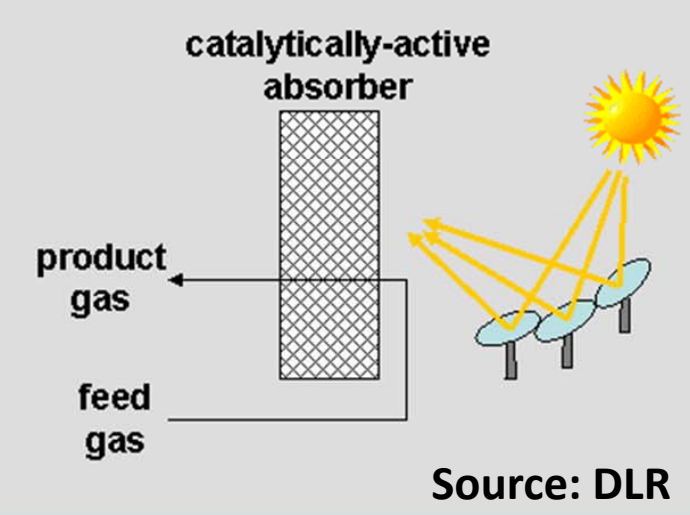

Reformer heated

externally $\left(700\right.$ to $\left.850^{\circ} \mathrm{C}\right)$

E.g. ASTERIX project
Irradiated reformer tubes (up Catalytic active direct to $850^{\circ} \mathrm{C}$ ), temperature gradient

Development: Australia, Japan; Research in Germany and Israel irradiated absorber

DLR coordinated projects: SOLASYS, SOLREF; Research in Israel, Japan

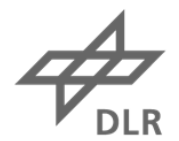




\section{Reforming vs. W/CD redox-oxides-"splitting" Chemistry}

- Employs fossil fuel $\left(\mathrm{CH}_{4}\right)$ as reactant.

- Solid catalyst: Ni-based catalysts supported on $\mathrm{CaAl}_{6} \mathrm{O}_{10}$ or $\mathrm{MgAl}_{2} \mathrm{O}_{4}$; noble metals ( $\mathrm{Ru}, \mathrm{Rh}, \mathrm{Pd}, \mathrm{Pt}) ; \mathrm{Fe}, \mathrm{Co}$.

- Temperature range: $700-850^{\circ} \mathrm{C}$.

- Gaseous reactants can be fed continuously.

- Employs $\mathrm{CO}_{2}$ as a reactant; i.e. can "reuse/valorize" atmospheric $\mathrm{CO}_{2}$.

- Solid redox-pair materials: ferrites $\left(\mathrm{NiFe}_{2} \mathrm{O}_{4}, \quad \mathrm{CoFe}_{2} \mathrm{O}_{4}\right), \quad \mathrm{CeO}_{2}-\mathrm{ZrO}_{2}$, perovskites $\left(\mathrm{La}_{1-\mathrm{x}} \mathrm{Sr}_{\mathrm{x}} \mathrm{Mn}_{\mathrm{y}} \mathrm{Al}_{1-\mathrm{y}} \mathrm{O}_{3-\delta}\right)$.

- Temperature range: $750-1500^{\circ} \mathrm{C}$.

- Solid is not a "catalyst" but a reactant, with non-negligible mass to be heated to the reaction temperature and progressively depleted during reaction, having to be replenished (reactions cannot be carried out continuously).

\section{Reforming vs. W/CD "splitting" solar reactors}

- Structured reactors.

- Solar heating: direct or indirect.
- Structured \& non-structured (particle) reactors.

- Solar heating: only direct (required Ts too high for indirect heating). 


\section{Principle of the solar thermal fuel production}

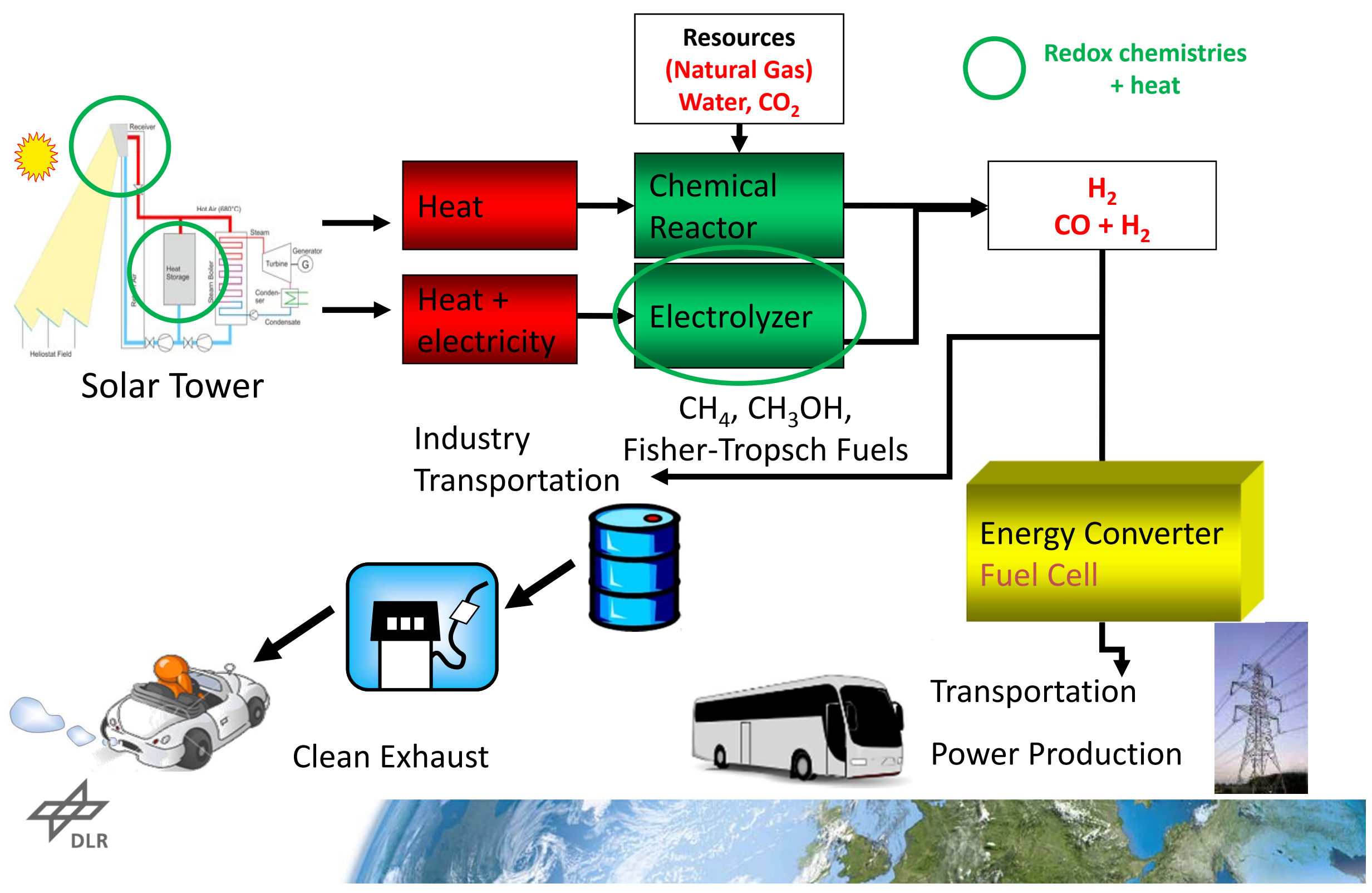




\section{Redox-oxide-based thermochemical cycles - structured} iveactors / heat exchangers

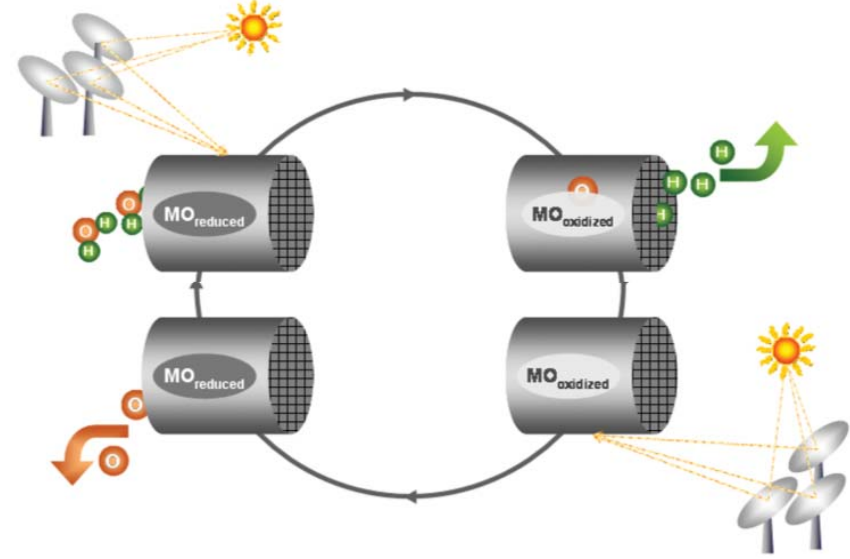

1st Step: Thermal reduction (Regeneration)

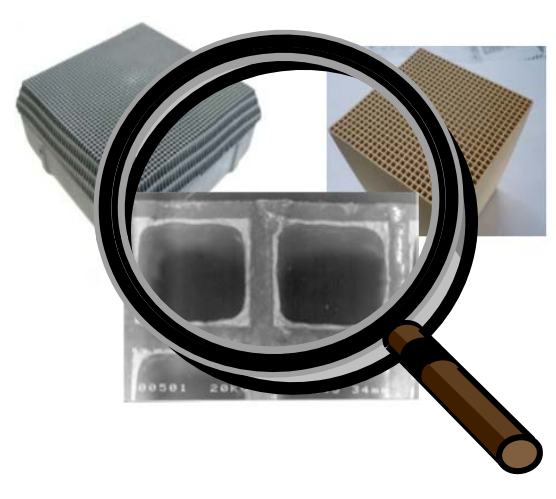

On Sun

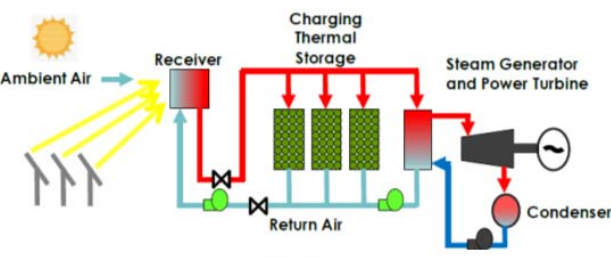

Off Sun

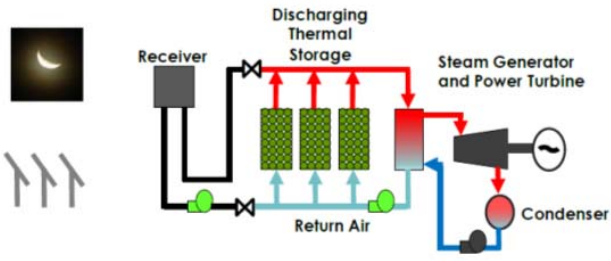

2nd Step: $\mathrm{H}_{2} \mathrm{O} / \mathrm{CO}_{2} \quad$ Splitting WS /CDS 2nd Step: (Air) Oxidation (AO)

$\mathrm{H}_{2} \mathrm{O}+\mathrm{MO}_{\text {red }} \rightarrow \mathrm{MO}_{\mathrm{ox}}+\mathrm{H}_{2}+(\Delta \mathrm{H})$

$\mathrm{CO}_{2}+\mathrm{MO}_{\text {red }} \rightarrow \mathrm{MO}_{\text {ox }}+\mathrm{CO}+(\Delta \mathrm{H})$

Net reaction: $\mathrm{H}_{2} \mathrm{O} \rightarrow \mathrm{H}_{2}+1 / 2 \mathrm{O}_{2}$

Net reaction: $\mathrm{CO}_{2} \rightarrow \mathrm{CO}+1 / 2 \mathrm{O}_{2}$

Net effect: Solar $\mathbf{Q} \rightarrow$ Solar Fuels

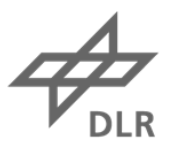

( $\mathrm{H}_{2}$, syngas)

$$
\mathrm{MO}_{\text {red }}+1 / 2 \mathrm{O}_{2} \rightarrow \mathrm{MO}_{\mathrm{ox}}+\Delta \mathrm{H}
$$

TR aided by electrical energy: (high T) Solid Oxide (co)electrolysis Cell (SOEC) for WS/CDS to $\mathrm{H}_{2} / \mathrm{CO}$.

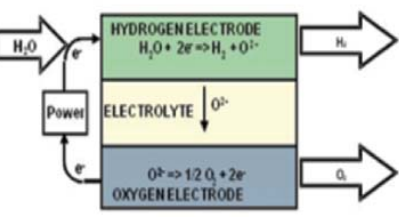

Net effect: Solar $Q \rightarrow \Delta H \rightarrow Q$ non-solar

Thermochemical storage 


\section{Solar receiver/reactor types (particles vs. porous solids; moving vs. non-moving parts)}
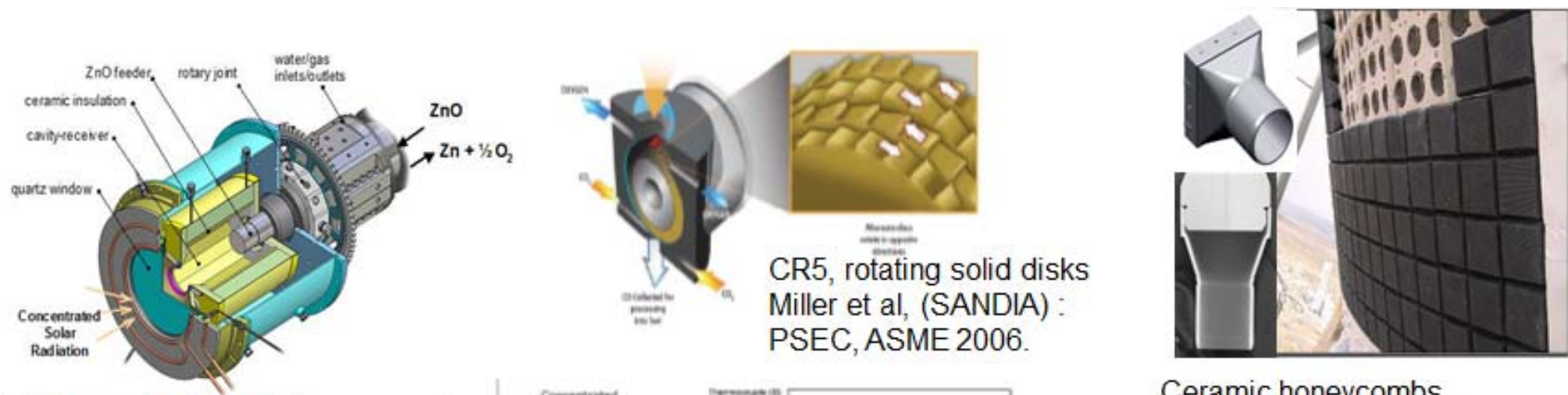

Rotating cavity (powder) Loutzenhiser et al (ETH), Materials, 2010.

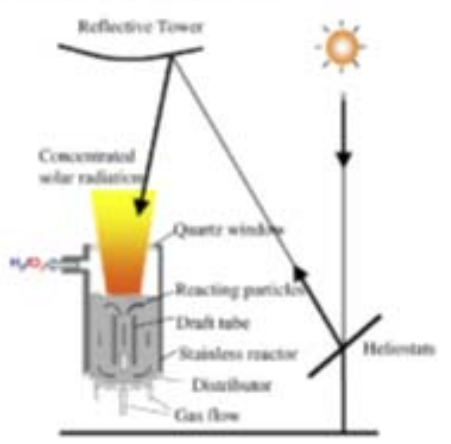

Spouted (powder) bed,

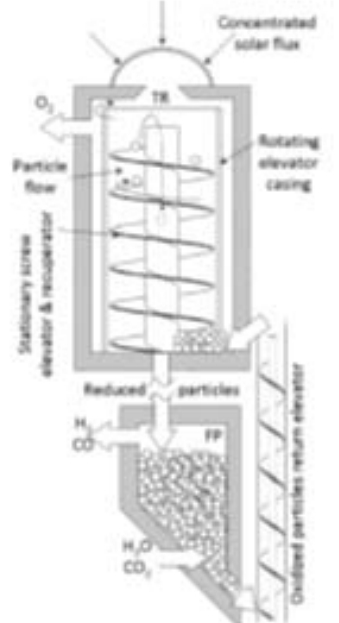
PSEC, ASME 2006. Gokon et al (Niigata Univ.) IntJHEn, 2011.

Moving powder bed

Ermanoski et al, (SANDIA)

JSolEnergyEng 2013.

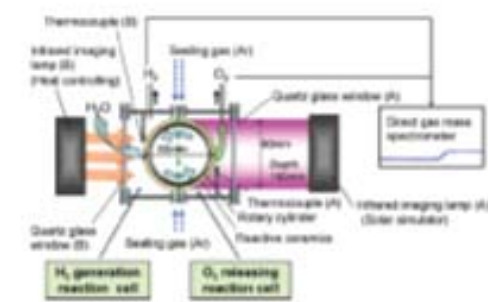

Rotating solid drum

Kaneko et al, (Tokyo Univ.), EnFuels 2007.
Ceramic honeycombs SOLAIR receiver (DLR), 2004.

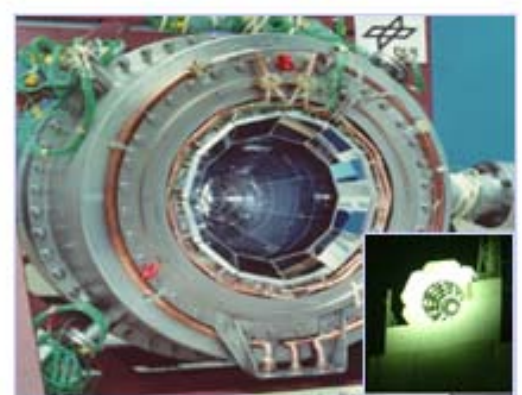

Ceramic foams SOLREF reactor (DLR), 2004.
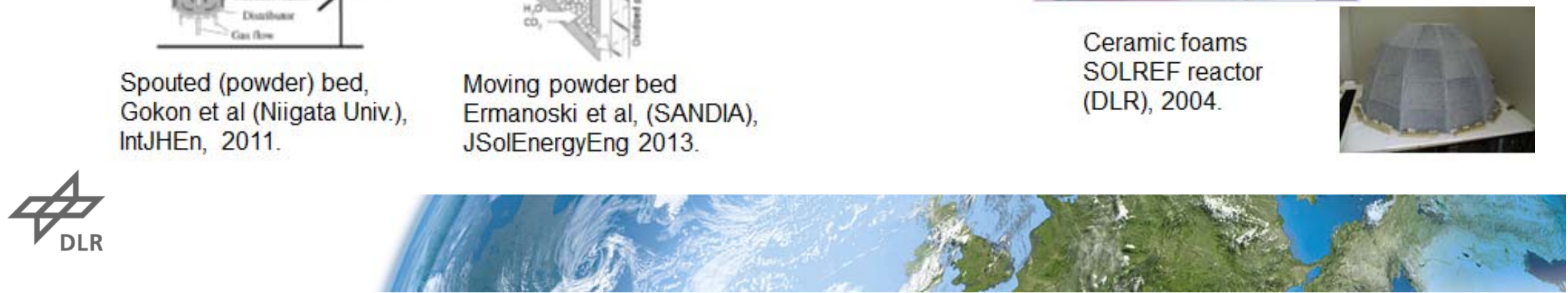


\section{Solar fuels: Solar receiver/reactors based on coated honeycombs:}

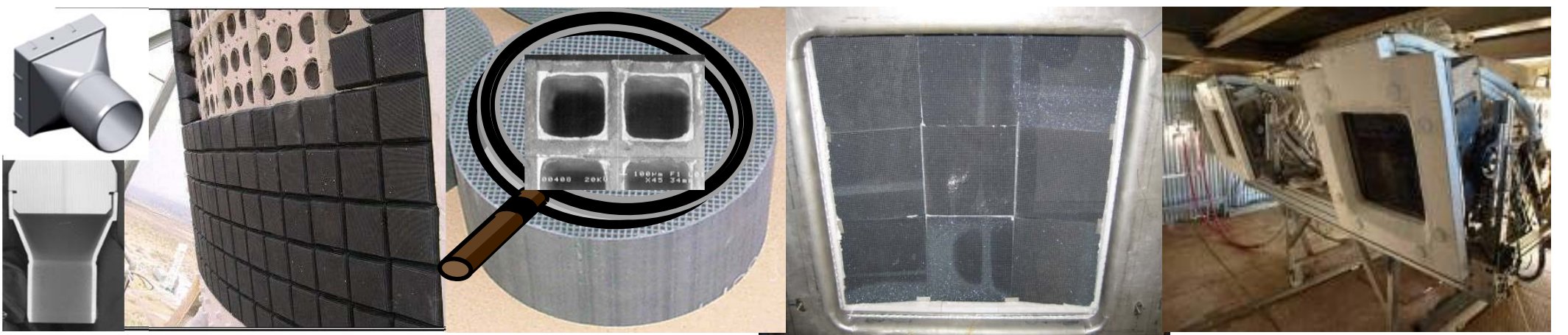

C. Agrafiotis, M. Roeb, A.G. Konstandopoulos, L. Nalbandian, V.T. Zaspalis, C. Sattler, P. Stobbe, A.M. Steele, Solar water splitting for hydrogen production with monolithic reactor, Solar Energy, 79(4), 409-421, (2005).

From active-material-coated "inert" structural supports to structures made entirely of the active material:

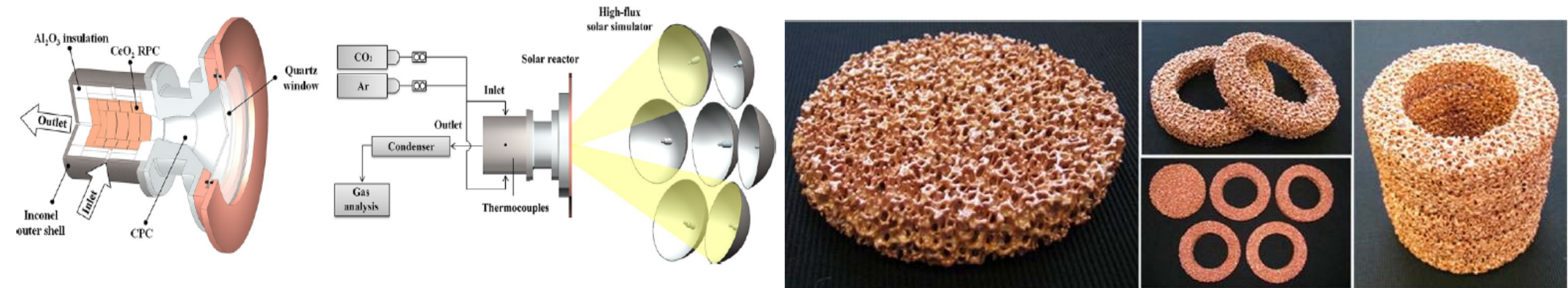

P. Furler, J. Scheffe, M.Gorbar, L. Moes, U. Vogt, A. Steinfeld, Solar Thermochemical $\mathrm{CO}_{2}$ Splitting Utilizing a Reticulated Porous Ceria Redox System, Energy \& Fuels, 26(11), 7051-59, (2012).
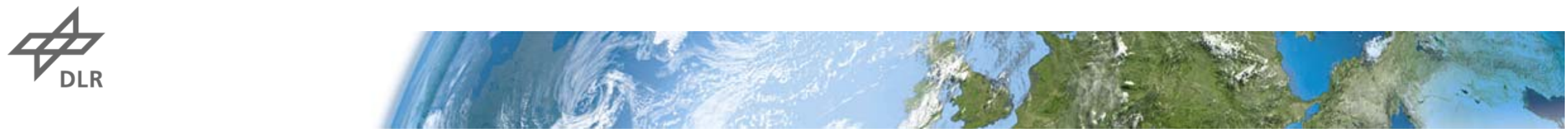


\section{Further scale-up: "Convergence" of reactor concepts}

Directly heated receiver/reformers (SOLASYS, SOLREF, 1998-2009)

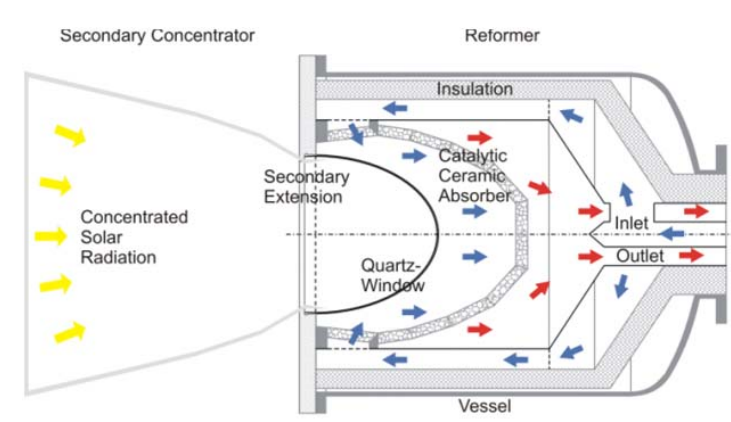

$\mathrm{Rh} / \mathrm{Al}_{2} \mathrm{O}_{3}$-coated

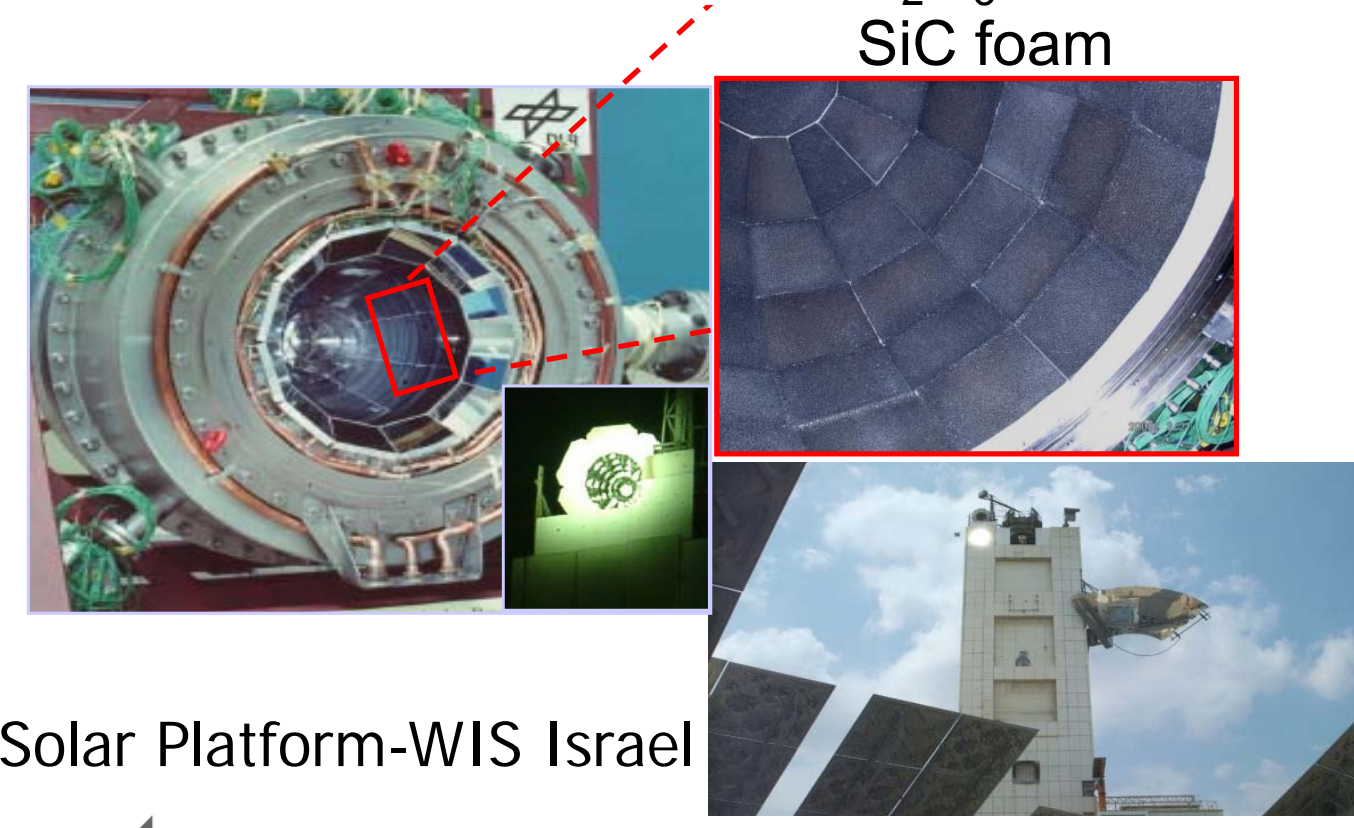

Directly heated WS/CDS reactors (HYDROSOL-PLANT, 2012-2017)

- Domed reactor chamber.

- Assembled of individual foam pieces.

-WS/CDS: "Redox-oxide-made" foams (from $\mathrm{NiFe}_{2} \mathrm{O}_{4}$ and $\mathrm{CeO}_{2}$ $\mathrm{ZrO}_{2}$ ); interchangeable with catalystcoated ones (SMR).

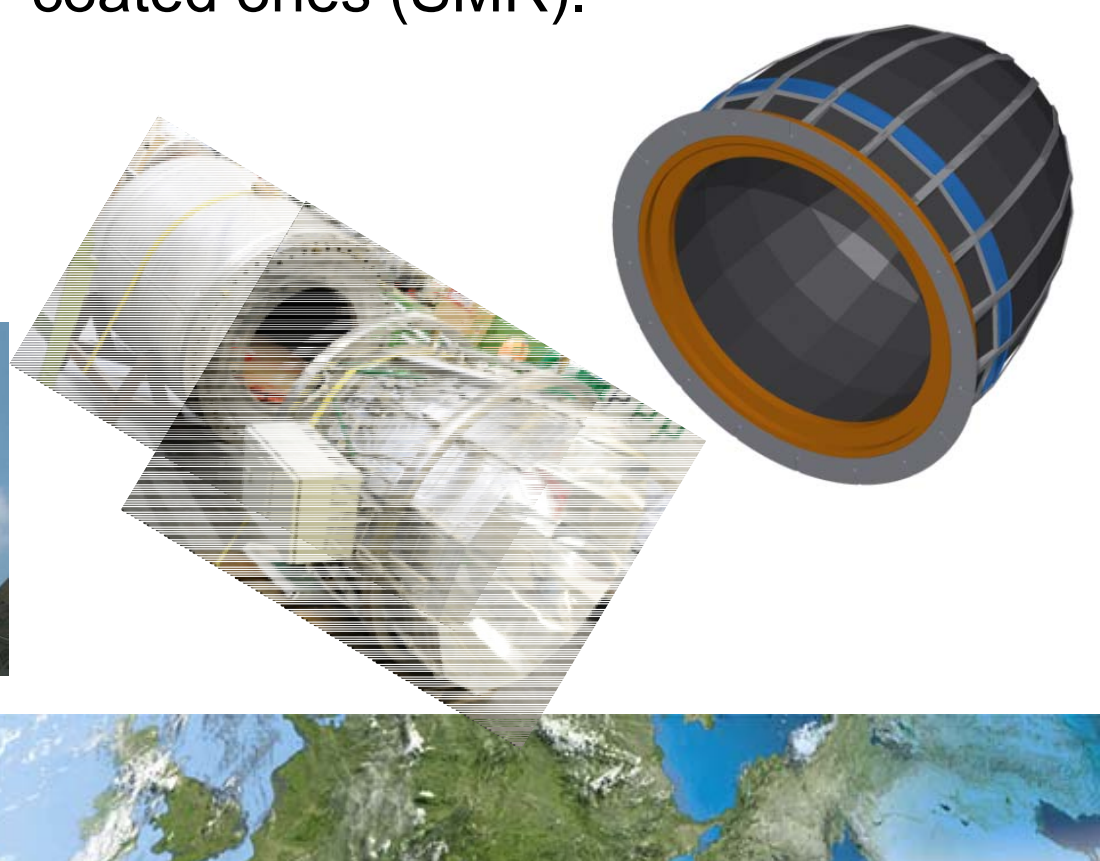




\section{HYDROSOL Technology: Continuous (dual chamber) Solar Receiver/ Reactor scalability and evolution}

2017: $750 \mathrm{~kW}_{\text {th }}$, Almeria, (schack et al. Solar Energy, 2016,17).

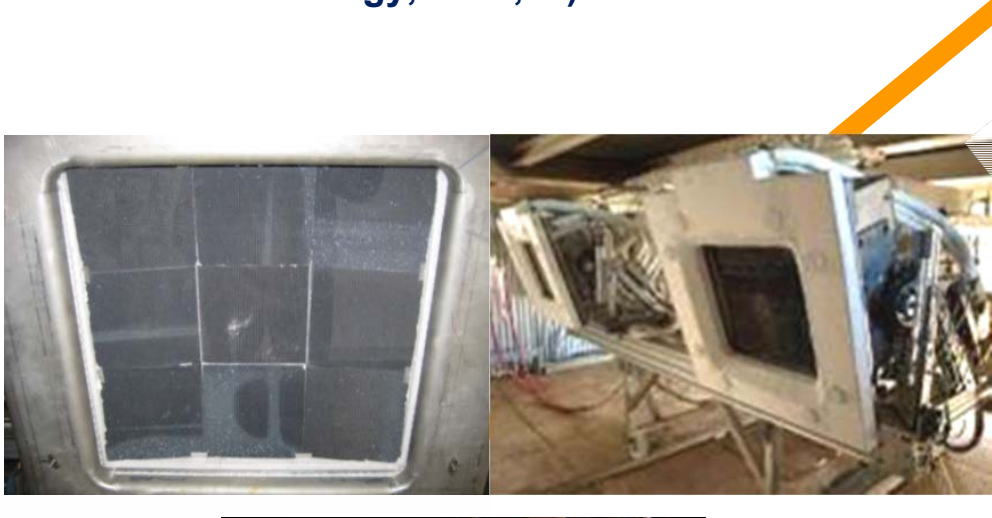

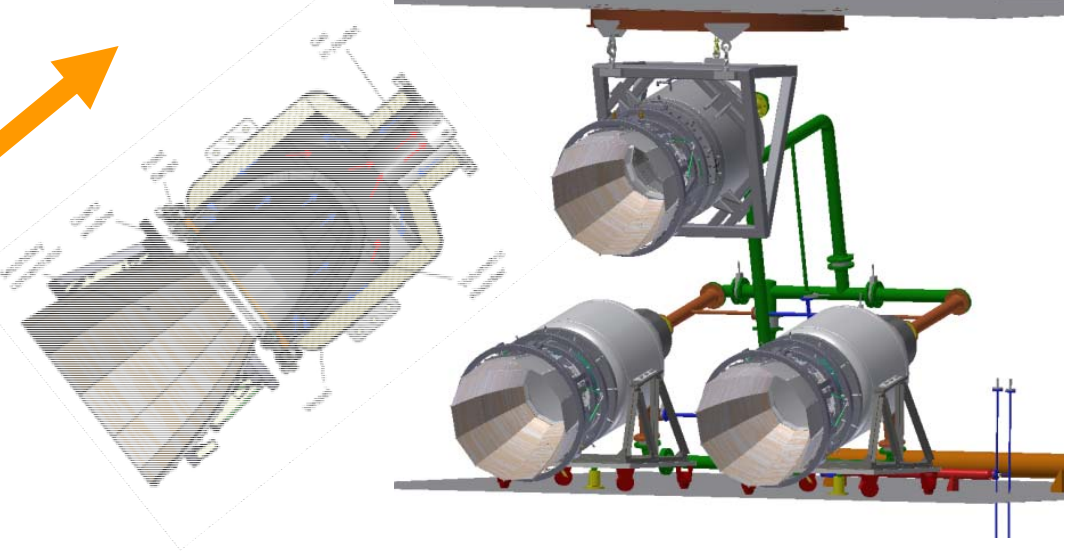
2008: 100 kW, PSA, Almeria, (Roeb et al, Solar Energy, 2011).

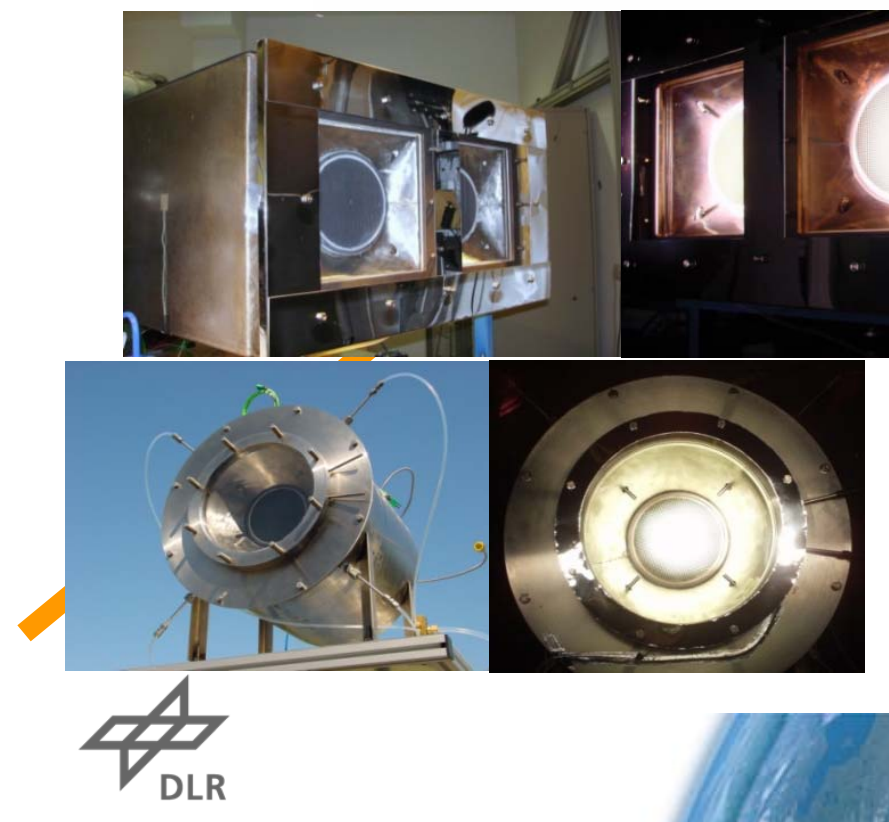

2004: 3 kW, DLR, Cologne, (Roeb et al, WHEC, 2006).

2002: $0.5 \mathrm{~kW}$, DLR, Cologne, (Agrafiotis et al, Solar Energy, 2005).
2017: 50 kW, ETH, Madrid, (Marxer et al, Energ. Envi. Sci,2017).
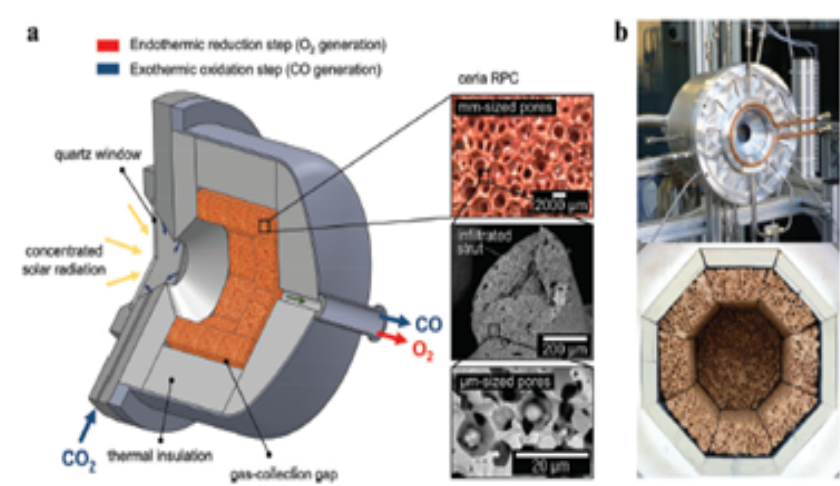

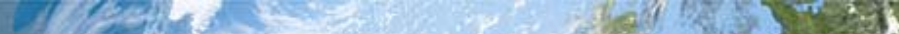




\section{From WS/CDS to TCS}

\section{(or from direct heating to allothermal heating)}
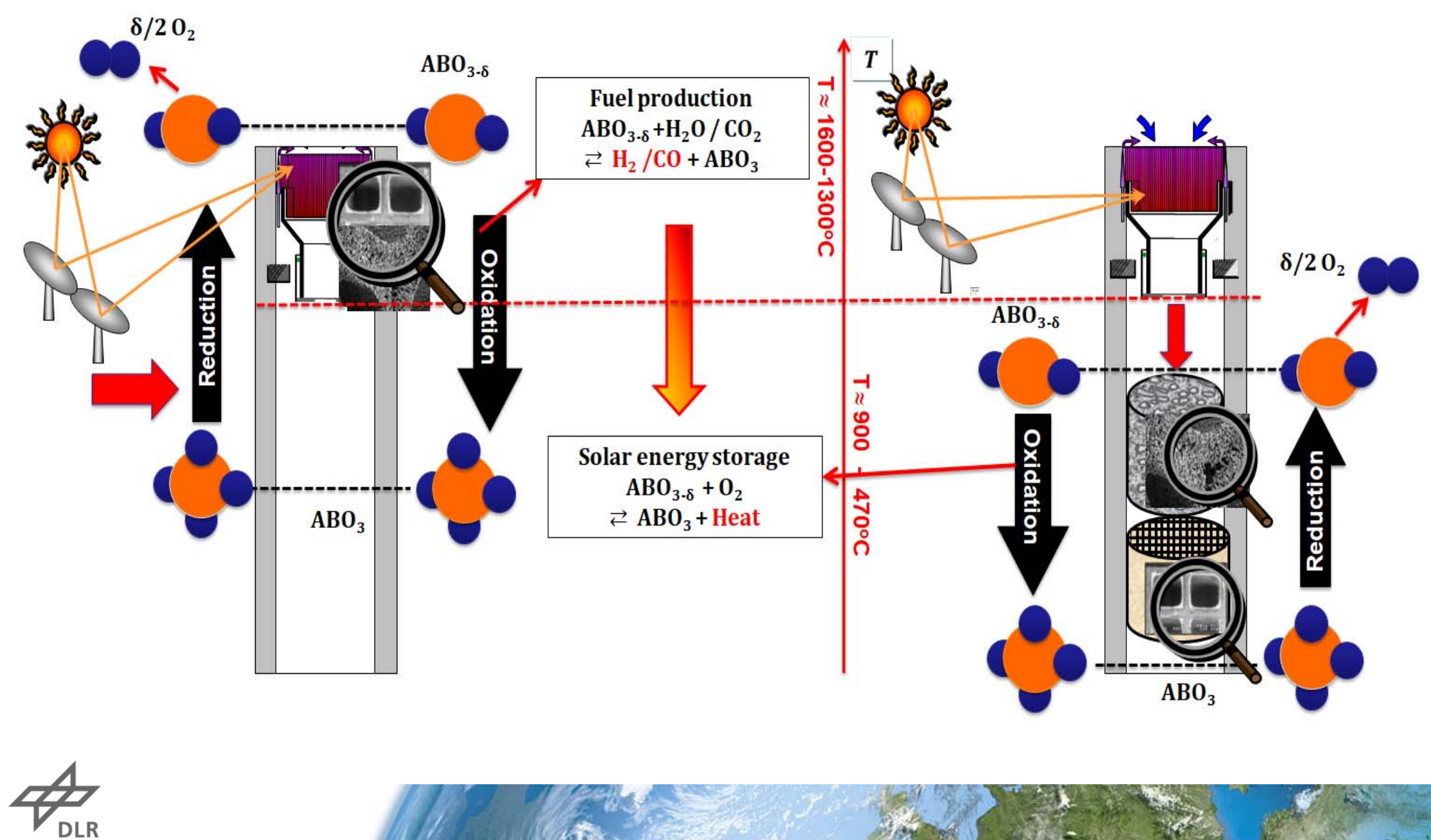


\section{RESTRUCTURE/ STOLARFOAM technology: TCS reactor/ heat exchanger scalability and evolution}

Lab-scale $\mathrm{CO}_{3} \mathrm{O}_{4}$-made and coated objects (Pagkoura et al, Solar Energy, 2014, Tescari et al, 2014. Karagiannakis et al, Solar Energy, 2016, Agrafiotis et al, Solar Energy 2014, 2015, 2016).

$\mathrm{m}_{\mathrm{C} 03 \mathrm{O} 4}=10-150 \mathrm{~g}$

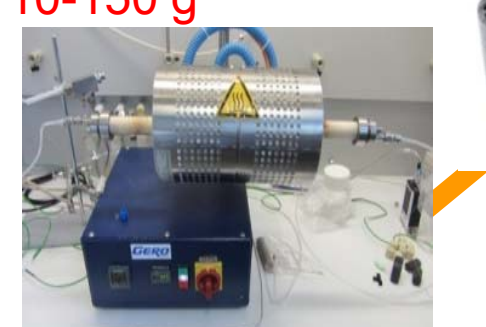

Powders
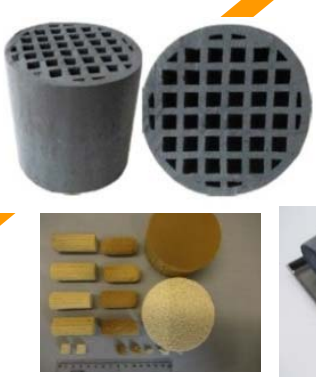

$\operatorname{mini} \mathrm{CO}_{3} \mathrm{O}_{4}$

$\mathrm{m}_{\mathrm{C} 0304}=200 \mathrm{mg}$ made and coated objects

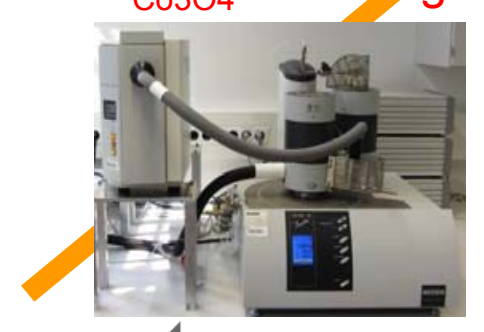
100 cycles; all $\mathrm{Co}_{3} \mathrm{O}_{4}$ exploited, no activity loss
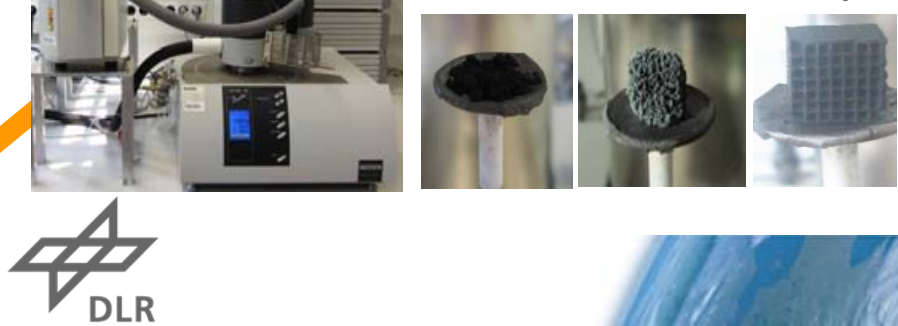

Pilot-scale $\mathrm{Co}_{3} \mathrm{O}_{4}$ coated cordierite honeycombs

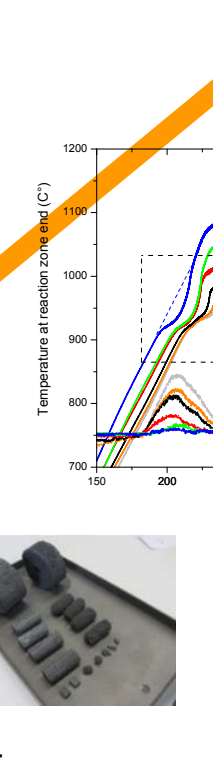

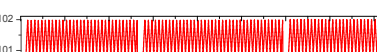

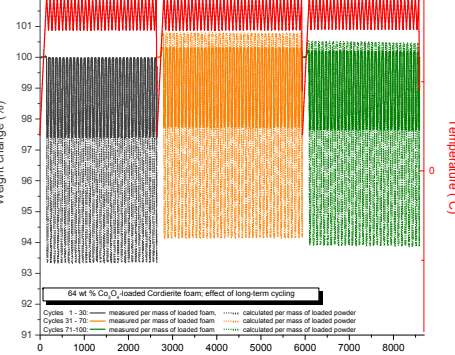

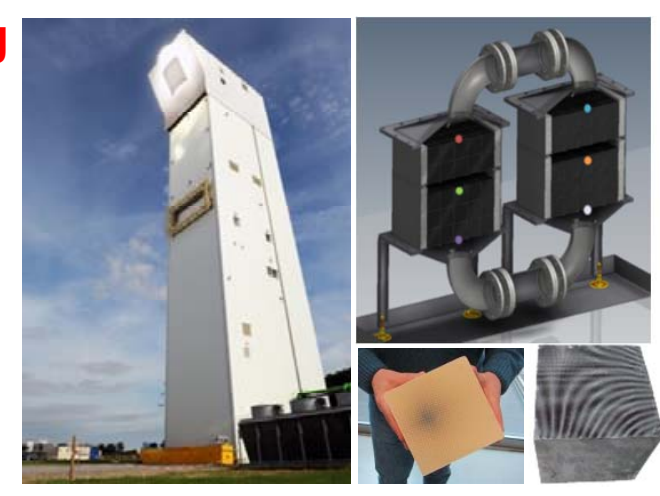

1

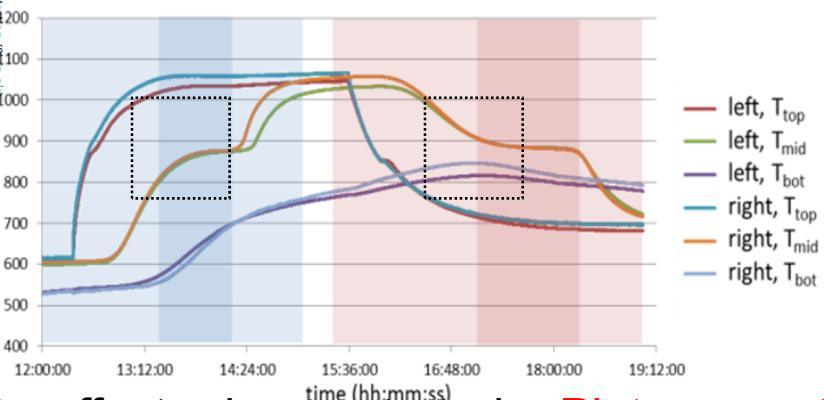

TCS effect demonstrated: Plateaus at constant temperature with $\mathrm{Co}_{3} \mathrm{O}_{4}$-coated honeycombs. High energy density; efficient heat release, cyclic performance without degradation over 15 cycles, structural integrity maintained, no coating spallation (Tescari et al, Applied Energy, 2017, Singh et al, Solar Energy, 2017). 


\section{Properties of merit required for redox oxide pairs}

WS/CDS TCS

\section{SOEC}

- Reduction of oxidized oxide state at "reasonable" temperatures

Under low $\mathrm{P}_{\mathrm{O} 2}$

- Reactivity of reduced oxide with $\mathrm{H}_{2} \mathrm{O} / \mathrm{CO}_{2}$

- High volumetric/ gravimetric $\mathrm{H}_{2}$, CO yield

\section{Under air}

- High $\Delta \mathrm{H}$ of air oxidation; reversibility

- High volumetric energy storage density
Under applied voltage

- High ionic (oxygen) and electronic conductivity

- Reactivity with $\mathrm{H}_{2} \mathrm{O}$ and/or $\mathrm{CO}_{2}$

- Long-term cycling chemical, mechanical, thermal and dimensional stability

WS/ CDS materials

Ferrites $(\mathrm{Ni}, \mathrm{Co}) \mathrm{Fe}_{2} \mathrm{O}_{4-\delta o x}$

Ceria $\mathrm{CeO}_{2-\delta o x}$

Perovskites: $\mathrm{La}_{1-\mathrm{x}} \mathrm{Sr}_{\mathrm{x}} \mathrm{AlO}_{3-\delta o x}$

$\mathrm{T} \approx 1500-700^{\circ} \mathrm{C}$
TCS materials

$\mathrm{Co}_{3} \mathrm{O}_{4} \mathrm{~T}_{\text {eq }}=870^{\circ} \mathrm{C}$

(Fe, Mn) ${ }_{2} \mathrm{O}_{3} \mathrm{~T}_{\mathrm{eq}}=970-920^{\circ} \mathrm{C}$

Perovskites:CaMn ${ }_{1-y} \mathrm{~B}_{\mathrm{y}} \mathrm{O}_{3-\delta o x} \mathrm{La}_{1-\mathrm{x}} \mathrm{Sr}_{\mathrm{x}} \mathrm{Co}_{\mathrm{y}} \mathrm{Fe}_{1-\mathrm{y}} \mathrm{O}_{3-\delta o x}$
$\mathrm{O}_{2}$ electrode materials

LSM-YSZ

Perovskites: LSCF 


\section{Criteria for solar thermal materials/processes selection?}

"... You can't always get what you want, but if you try, sometimes you just might find, you get what you need...". The Rolling Stones, 1969.

\section{Want!}

CSP-carbon-neutral solar fuels from sun, $\mathrm{H}_{2} \mathrm{O}$ and $\mathrm{CO}_{2}$ but "...the reactions involved are on the edge of being feasible and practicable...".

CSP-reactors with high theoretical efficiency.

Redox pair material compositions that can be thermally reduced and split $\mathrm{H}_{2} \mathrm{O} / \mathrm{CO}_{2}$.

\section{Need?}

Hybrid options exploiting similar materials and reactors yet realizable under milder conditions, as a transition path from fossil fuel-based solar-fuels to such produced only by renewable resources?

Technically simpler, viable, pragmatic CSP-reactor concepts attractive for largescale implementation and demonstration?

Bulk, robust, porous oxide structures from inexpensive raw materials, that can perform cyclic redox operations for extended periods of time? (WS/CDS, TCS, Membranes, SOECs)? 
Thank you for your attention!

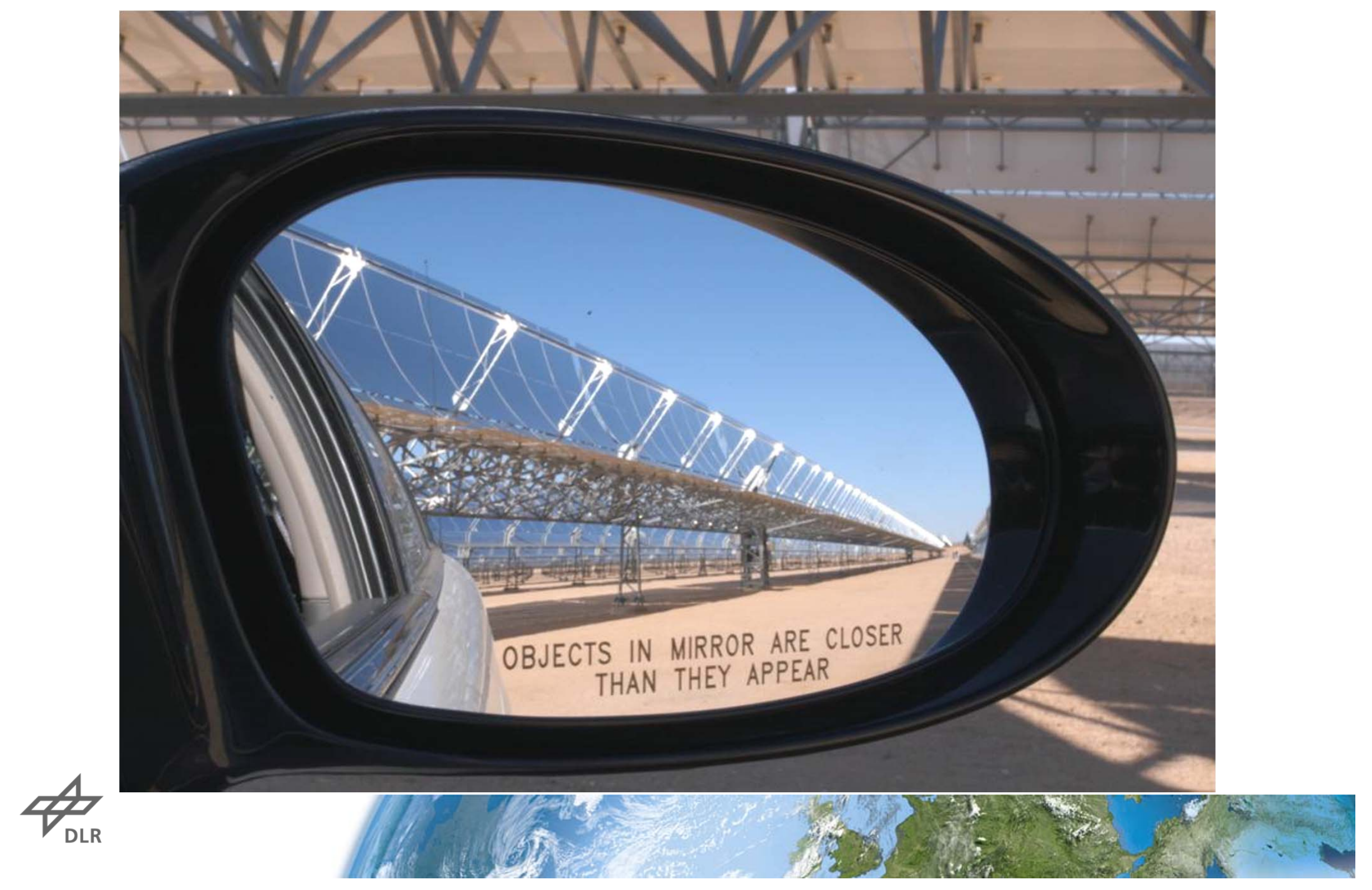

